\title{
A Random Walk Model of Wave Propagation
}

\author{
Massimo Franceschetti, Member, IEEE, Jehoshua Bruck, Fellow, IEEE, and Leonard J. Schulman
}

\begin{abstract}
This paper shows that a reasonably accurate description of propagation loss in small urban cells can be obtained with a simple stochastic model based on the theory of random walks, that accounts for only two parameters: the amount of clutter and the amount of absorption in the environment. Despite the simplifications of the model, the derived analytical solution correctly describes the smooth transition of power attenuation from an inverse square law with the distance to the transmitter, to an exponential attenuation as this distance is increased-as it is observed in practice. Our analysis suggests using a simple exponential path loss formula as an alternative to the empirical formulas that are often used for prediction. Results are validated by comparison with experimental data collected in a small urban cell.
\end{abstract}

Index Terms-Microcellular systems, path loss, random media, random walks, wave propagation.

\section{INTRODUCTION}

$\mathbf{R}$ ADIO microcells for wireless personal communication have become increasingly popular in the last few years, and today many telecommunication companies use them to provide coverage to densely populated areas. Internet service providers have also started using them to provide high-speed wireless internet access. These microcells are regions of few hundred meters of diameter, served by radio base stations. They are one or two order of magnitude smaller than traditional radio cells used for personal communication. The reduction in size requires the use of low antennas (typically below $10 \mathrm{~m}$ ), transmission at low power (typically below $10 \mathrm{~W}$ ), and has the advantage of increasing the overall capacity of the system. Another effect of using low transmitting antennas is that the propagation mechanism is dominated by the large amount of scattering and diffraction due to the many objects encountered by the radiated signal. Lamp posts, street signs, trees and vegetation, pedestrians, cars, irregularly sited and textured building walls (common in older European constructions), can scatter energy in many directions, allowing reception of signal in shaded areas. These effects are difficult to predict, and exact analytical solutions, obtained by solving Maxwell equations in the given environment, cannot be sought for. The alternative is to develop simplified theoretical models that are analytically

\footnotetext{
Manuscript received February 17, 2003; revised May 30, 2003. This work was supported in part by the Caltech Lee Center for Advanced Networking, by the National Science Foundation under CAREER Grant 0049092 (previously 9876172), and by the Charles Lee Powell Foundation.

M. Franceschetti is with the Department of Electrical Engineering and Computer Sciences, University of California at Berkeley, Berkeley, CA 94720-1770 USA (e-mail: massimof@EECS.berkeley.edu).

J. Bruck is with the Department of Electrical Engineering, California Institute of Technology, Pasadena, CA 91125 USA.

L. J. Schulman is with the Department of Computer Science, California Institute of Technology, Pasadena, CA 91125 USA.

Digital Object Identifier 10.1109/TAP.2004.827540
}

tractable and that show good agreement with experimental findings.

The main contribution of this paper is to show that a reasonably accurate description of the propagation loss in microcells can be obtained adopting a very simple model. We stochastically describe the propagation environment using only two parameters: the amount of clutter and the amount of absorption in the environment. Then we analytically derive a power loss formula using the theory of continuous random walks [20]. Our model is a great oversimplification of the real physics of propagation. We are not interested in a very accurate description of all the features of the built up terrain, that would require over-fitting the model with a large number of parameters. On the contrary, we pursue the idea that a very simplified model can still make good predictions and explain physical phenomena that are observed in practice. Accordingly, we validate our results by comparison with experimental data and we propose a simple exponential attenuation formula, based on the theoretical results, that can be used for practical calculation purposes. This simple formula has been recently used in [31] to derive new bounds on the capacity of wireless multihop networks. Our model may not fit areas with high rise buildings and transmitting antennas on the roofs. However, there is a large class of built up areas where the model is appropriate: even in a large city (illuminated by a low antenna) experiments validated our theory.

The paper is organized as follows. In the next section we describe how previous work on modeling propagation has followed fundamentally different schools of thought, and we place our approach in context. In Section III, we introduce the random walk model and derive some probabilistic quantities of interest. In Section IV, we apply the random walk model to derive two physical quantities: the radiated power density in the radial direction, i.e., the amplitude of the radial component of the Poynting vector $S(r)$; and the full power density, obtained by the (power) superposition of all waves crossing an elementary area from all directions. This latter quantity is of interest in cluttered environments, where waves may reach the observation point in principle from any direction. In Section V, we show relationships with the classical theory of wave propagation in random environments. In Section VI, we introduce the radio link equation for cluttered environments. Comparison with experimental data is provided in Section VII. Conclusions are drawn in Section VIII.

\section{DifFERENT SchoOls OF ThOUght}

Previous work modeling wireless propagation has emphasized very different approaches. On one side, a simple power-law decay law with the distance to the transmitter has been considered, for both line of sight (LOS) and non line of 
sight (NLOS) conditions. This inverse power law model goes back to the empirical formula given by Hata [19], following previous work of Okumura et al. [26], based on extensive experimental measurements made in the nineteen-sixties in Japan. When adapting the Okumura-Hata model to microcellular systems, there is experimental evidence of an inverse power law exponent that increases as a function of the distance between the transmitter and the receiver. To accommodate to this variation, telecommunication companies started using path loss models characterized by a breakpoint distance at which the exponent of the inverse power law is changed from a value close to 2 , to a value in the range of 4 to 10 . These high order power laws, after a breakpoint, have been consistently reported in the literature [7]-[10], [16]. Power law models also have a theoretical justification in simplified two and four ray interfering models, and multiple diffraction models, but these can explain power exponents only up to 4.5 [2], [22].

A second approach has been ray tracing [27]-[29]. In this case, one trades the simplicity of a simple power law for the complexity of an accurate numerical solution. Ray tracing assumes that the location and the reflection properties of all important objects inhibiting propagation are accurately known, and then calculates the interfering effects of the different wave paths at the receiver. Usually, it assumes that the geometrical optics limits of electromagnetic wave propagation are adequate, but more realistic models accounting for the roughness of building walls, decomposing them into smaller patches that provide scattering in all directions, have also been proposed [30].

A third approach is stochastic in nature and represents a tradeoff between the empirical power laws interpolating experimental data, and the accuracy of numerical simulations obtained by ray tracing. In this case, the environment is modeled probabilistically, using a few parameters, and analytical formulas are derived. One of these models is the multislit waveguide [3], [5], [24] that applies to LOS conditions of propagation along a straight street with randomly distributed walls of buildings and gaps between them. This model takes into account many parameters, like the street width, the mean distribution of the buildings along the street, electrical properties of the building walls, height and placement of the transmitting antenna. It predicts a smooth transition from an $r^{-2}$ mode to an exponential attenuation mode that can explain the empirical high order power law of $r^{-q}, q>4$, obtained in most experiments in LOS conditions. A similar stochastic model for NLOS propagation has been proposed in [4], [6]. This is also a multiparametric model that uses up to six parameters to give an accurate description of the features of the build up terrain and of the propagation mechanism.

A completely different philosophy is represented by the stochastic approach presented in [12] and [23]. The objective of these works is to have a model with the fewest number of parameters that can still give some global information of the field properties. The authors propose to model an urban area using only one parameter, that is the density of the (lossless) obstacles in the environment. They consider a random walk formulation of the problem of optical ray penetration inside the medium. The main result is a closed form formula for the propagation depth of a wave entering the medium at a given angle. Their study, however, does not consider signal attenuation, and is limited to propagation in two dimensions, through obstacles arranged in a Manhattan-type grid pattern. In this paper, we follow the same philosophy of adopting a simple model that can explain real physical phenomena, but we remove the above limitations, considering an isotropic source placed inside the environment and including signal attenuation and losses due to absorption. The model is solved analytically and a path loss formula is obtained. When compared with experimental data, the formula correctly describes the smooth transition (already noted in [5] for the LOS case of the multislit waveguide model) of power attenuation from the free space mode $r^{-2}$ to an exponential attenuation, and is, in the medium range, close to the empirical formulas that use high order power laws, after a breakpoint, to fit experimental data.

\section{The WANDERING PHOTON}

Consider free space filled with a uniform random distribution of scattering objects. At the origin in this cluttered environment a source radiates a monochromatic isotropic wave of angular frequency $\omega$. The received signal will strongly fluctuate, due to the random superposition of many echoes from the scatterers: the superposition of the received signals with random phases and polarizations provides a fast space-time varying signal, whose average, over an interval of some wavelengths, or few periods $2 \pi / \omega$, is zero (or close to zero). To filter out these strong oscillations in the received signal, one can measure the received power, which can be theoretically computed by incoherent summation of the received echo's powers, and practically evaluated by averaging the received power over time intervals of several signal periods, or space intervals of several signal wavelengths. Modeling the radiating wave as a stream of photons propagating in the environment, this corresponds to summing the intensity of all different waves reaching the receiver. In our model each radiating photon proceeds along a straight line for a random length, until it hits an obstacle. The photon is then either absorbed by the obstacle, with probability $\gamma$, or it is scattered in a random direction, with probability $1-\gamma$. In this way, a photon propagates in the environment along a random piecewise linear trajectory, (see [20] for background on this subject), as in Fig. 1.

Let $Q(r)$ be the probability density function (pdf) of hitting an obstacle at distance $r$ from the photon's source, at the first step of the random walk. The pdf $G(r)$ of the photon's absorption, at distance $r$ from the source, can be obtained by solving the following:

$$
G(r)=\gamma Q(r)+(1-\gamma) Q * G(r)
$$

where the symbol "*" stands for convolution. Use of (1) can be justified as follows. Let us iteratively substitute the expression for $G(r)$ in the convolution integral. We have $G(r)=$ $g_{0}(r)+g_{1}(r)+g_{2}(r)+\cdots$, with $g_{0}(r)=\gamma Q(r), g_{1}(r)=(1-$

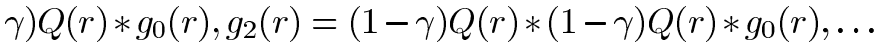
In this recursive series $g_{0}(r)$ describes the event that the photon is absorbed at the first step of the random walk, hitting a single obstacle; $g_{1}(r)$ describes the event that the photon is absorbed 


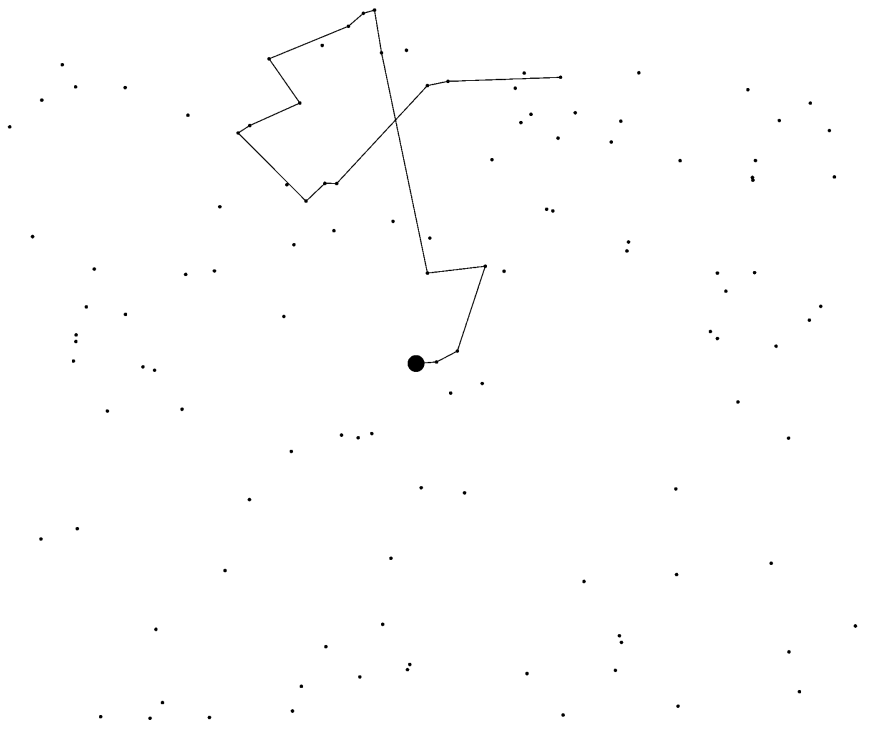

Fig. 1. Sketch of the propagation model. Each photon radiating from an isotropic source propagates in the environment following a piecewise linear trajectory, modeled as a random walk. Each time the photon hits an obstacle, it turns in a random direction, or it is absorbed by the obstacle.

at the second collision; and so on. Since all these events are disjoint, we sum all the $g_{i}$ 's to obtain the overall $\operatorname{pdf} G(r)$ of the photon's absorption at distance $r$ from the origin.

Equation (1) can be easily solved in the Fourier domain. Let

$$
g(\omega)=\mathrm{FT}[G(r)], \quad q(\omega)=\mathrm{FT}[Q(r)]
$$

be the Fourier transforms (FT) of $G(r), Q(r)$, respectively. It follows that

$$
g(\omega)=\frac{\gamma q(\omega)}{1-(1-\gamma) q(\omega)}
$$

so that

$$
G(r)=\mathrm{FT}^{-1}\left[\frac{\gamma q(\omega)}{1-(1-\gamma) q(\omega)}\right]
$$

We now specify the pdf $Q(r)$. Assuming the obstacles to be uniformly distributed according to a Poisson point process, the interobstacle hitting distance is an exponential random variable, and we have

$$
\begin{aligned}
& Q_{1}(r)=\frac{\eta e^{-\eta r}}{2} \\
& Q_{2}(r)=\frac{\eta e^{-\eta r}}{2 \pi r} \\
& Q_{3}(r)=\frac{\eta e^{-\eta r}}{4 \pi r^{2}}
\end{aligned}
$$

in one-, two-, and three-dimensions (1-D, 2-D, and 3-D), respectively. Note that $1 / \eta$ is the mean free path of the photon between successive collisions, so that $\eta$ is an estimate of the density of the obstacles in the environment. Note also that (4) is a pdf that integrates to one over the line; similarly (5) integrates to one over the plane; and (6) integrates to one over the whole space.
From a practical point of view, the most interesting case is 3-D, although 2-D is also relevant for very open terrestrial environments. We work out solutions for $G(r)$ in all three dimensions. Substituting expressions for FT $[Q(r)]$ into (3), we have

$$
\begin{aligned}
G_{1}(r)= & \frac{\sqrt{\gamma} \eta}{2} e^{-\eta \sqrt{\gamma} r} \\
G_{2}(r) \approx & \frac{\gamma \eta}{2 \pi r}\left[e^{-\left(1-(1-\gamma)^{2}\right) \eta r}\right. \\
& \left.+(1-\gamma) \eta r K_{0}\left(\sqrt{1-(1-\gamma)^{2}} \eta r\right)\right] \\
G_{3}(r) \approx & \frac{\gamma \eta}{4 \pi r^{2}}\left[(1-\gamma) \eta r e^{-\sqrt{1-(1-\gamma)^{2}} \eta r}+e^{-\left(1-(1-\gamma)^{2}\right) \eta r}\right]
\end{aligned}
$$

in 1-D, 2-D, and 3-D, respectively. In (7)-(9), $K_{\nu}(\cdot)$ is the modified Bessel function of the second kind. Equation (7) is derived without approximation and provides an elegant prediction: at any fixed level of clutter in the environment, the effective radius of the signal scales as the square root of $1 / \gamma$, the expected number of steps taken by the random walk. This phenomenon is closely related to the square-root-of-time scaling of the distance travelled by a symmetric random walk; however, the actual distributions in the two processes are quite different, being in this case a two-sided exponential rather than a normal distribution. Derivations leading to (8) and (9) are shown in Appendixes A and $\mathrm{B}$. In spite of the approximations underlying their derivation, all equations above integrate to unity, see Appendix A and $\mathrm{B}$, and so they are probability densities. An additional check shows that in all three cases, as $\gamma \rightarrow 1$ (perfectly absorbing obstacles), $G(r)$ tends to $Q(r)$, as the random walk reduces to a single exponentially distributed step. Finally, in Appendix A we also show an exact derivation for the 2D case, expressing $G(r)$ in terms of an infinite series of Bessel polynomials.

\section{From Probability to Physics}

We now apply the probabilistic results obtained in the previous section to compute two physical quantities of interest: the radiated power density and the full power density at a given distance from the transmitter. Let us refer to the 3-D case for their definitions.

We first consider the power flux in the radial direction, that is obtained by integration of $G(r)$ over the region outside $r$, which gives all the photons of the radiating wave that are absorbed outside a sphere of radius $r$ centered at the transmitter. The radiated power density at distance $r$ is obtained by normalizing the power flux by the surface of the sphere. Note that this corresponds to calculating the expected value of the Poynting vector intensity $S(r)$. The other quantity of interest is the full power density, represented by all the photons of the radiating wave that enter an elementary sphere of radius $\Delta r$, placed at distance $r$ from the source, normalized to the sphere surface, and in the limit for $\Delta r \rightarrow 0$. Note that this latter quantity takes into account the power that is reflected toward the observation point from all directions.

In free space, or when obstacles are perfectly absorbing, the full power density and the radiated power density coincide, because all the power propagates in the radial direction; but the 
presence of reflecting obstacles can provide radiation from different directions and the two quantities become different. This effect is more pronounced as $\gamma \rightarrow 0$ and propagation becomes diffusive [21].

\section{A. Radiated Power Density}

We start with the computation of $S(r)$. In 1-D we have

$$
S(r)=\int_{r}^{\infty} G(r) d r=\frac{e^{-\eta \sqrt{\gamma} r}}{2} .
$$

In the limit of few obstacles $(\eta \rightarrow 0)$ or low absorption $(\gamma \rightarrow$ $0), S(r) \rightarrow 1 / 2$ as the power splits evenly along the two directions of the line.

In 2-D we have

$$
\begin{aligned}
S(r)= & \frac{1}{2 \pi r} \int_{0}^{2 \pi} d \phi \int_{r}^{\infty} r G(r) d r \\
= & \frac{1}{2 \pi r}\left[\frac{1}{2-\gamma} e^{-\left(1-(1-\gamma)^{2}\right) \eta r}\right. \\
& \left.+\frac{\sqrt{\gamma} \eta(1-\gamma)}{\sqrt{2-\gamma}} r K_{1}\left(\sqrt{1-(1-\gamma)^{2}} \eta r\right)\right]
\end{aligned}
$$

where we have exploited identity 5.52 .1 in [15].

It is interesting to check the two limiting cases. When there are no obstacles $(\eta \rightarrow 0)$ we have, letting $z=\sqrt{1-(1-\gamma)^{2}} \eta r$

$$
\begin{aligned}
S(r) & =\frac{1}{2 \pi r}\left[\frac{1}{2-\gamma}+\frac{\sqrt{\gamma} \eta(1-\gamma)}{\sqrt{2-\gamma}} r K_{1}(z \rightarrow 0)\right] \\
& =\frac{1}{2 \pi r}\left(\frac{1}{2-\gamma}+\frac{1-\gamma}{2-\gamma}\right)=\frac{1}{2 \pi r},
\end{aligned}
$$

this is exactly what we expect for 2-D free space propagation of a cylindrical wave, having used the series expansion 8.446, of [15], for $K_{1}(z)$. Similarly, when there is no absorption $(\gamma \rightarrow 0)$, we have

$$
S(r)=\frac{1}{2 \pi r}\left(\frac{1}{2}+\frac{1}{2}\right)=\frac{1}{2 \pi r}
$$

which is what we expect by conservation of power.

In 3-D we have,

$$
\begin{aligned}
S(r)= & \frac{1}{4 \pi r^{2}} \int_{0}^{2 \pi} d \phi \int_{0}^{\pi} \sin \theta d \theta \int_{r}^{\infty} r^{2} G(r) d r \\
= & \frac{\gamma \eta}{4 \pi r^{2}}\left(\eta(1-\gamma) \int_{r}^{\infty} r e^{-\sqrt{1-(1-\gamma)^{2}} \eta r} d r\right. \\
& \left.+\int_{r}^{\infty} e^{-\left(1-(1-\gamma)^{2}\right) \eta r} d r\right) \\
= & \frac{1}{4 \pi r^{2}} \frac{1}{(2-\gamma)}\left[(1-\gamma)\left(\sqrt{1-(1-\gamma)^{2}} \eta r+1\right)\right. \\
& \left.\times e^{-\sqrt{1-(1-\gamma)^{2}} \eta r}+e^{-\left(1-(1-\gamma)^{2}\right) \eta r}\right] .
\end{aligned}
$$

It is again interesting to check the two limiting cases. We immediately see that when there are no obstacles $(\eta \rightarrow 0)$, or when there is no absorption $(\gamma \rightarrow 0)$, we have $S(r)=1 /\left(4 \pi r^{2}\right)$. Again this is what we expect on physical grounds.

\section{B. Full Power Density}

We want an expression accounting for all the photons of the radiating wave that enter an elementary sphere of radius $\Delta r$, placed at distance $r$ from the source, normal to the sphere surface, and in the limit for $\Delta r \rightarrow 0$. This is immediately related to $G(r)$ that represents the number of photons being absorbed inside the elementary sphere. Dividing $G(r)$ by the absorption coefficient $\gamma$ we obtain the number of photons intercepted by the obstacles inside the sphere, that are either absorbed or scattered in a random direction. Note that this number does not represent all the photons entering the sphere, but only the portion that undergoes a collision inside it. Now, consider a wave composed by a stream of photons proceeding along a straight line and entering the sphere along a given direction. In our model, on the average only one of every $1 /(\eta \Delta r)$ of these photons undergoes a reflection inside the sphere. Hence, to compute the number of photons that enter the sphere, and are either absorbed, scattered, or pass through undisturbed, we need to multiply by a factor of $1 / \eta$. It follows that the full power density is given by

$$
P(r)=\frac{G(r)}{\gamma \eta} .
$$

Note that the units of the full power density are [Watts $/ \mathrm{m}]$ and [Watts $\left./ \mathrm{m}^{2}\right]$ in 2-D and 3-D respectively, as expected. Moreover, on physical grounds we expect in free space the full power density to coincide with the radiated power density and to attenuate as $1 /\left(4 \pi r^{2}\right)$ in $3-\mathrm{D}$, and as $1 /(2 \pi r)$ in 2 -D. This can be seen by substituting $\eta \rightarrow 0$ into (12). The other limiting case when the radiated and full power densities become the same is when obstacles are completely absorbing $(\gamma \rightarrow 1)$. In this case, the attenuation law becomes $e^{-\eta r} /\left(4 \pi r^{2}\right)$ in 3-D and $e^{-\eta r} /(2 \pi r)$ in 2-D, which agrees with the classical theory (see [21, Chapter 12]). Finally, a cluttered environment with no absorption $(\gamma \rightarrow 0)$ leads to a diffusive regime, as is discussed in the next section.

\section{DIFFUSION IN RANDOM MEDIA}

The obvious starting point to characterize propagation in a wireless channel would be to use Maxwell equations. In principle, these can provide a complete characterization of the propagation loss in any given medium. As already noted, in the case of a rich scattering environment such as an urban area, this approach does not lead to closed-form solutions. An alternative approach is to treat the city as a random environment, and consists in studying propagation in a random medium. There is a huge literature on propagation in random media which dates back to the beginning of last century, with the study of radiation of light in foggy atmospheres. Historically, the problem has been investigated from two distinct points of view. One is "radiative transfer theory" or "transport theory," and the other is "multiple scattering theory" or "analytical theory." Radiative transfer theory is heuristic and deals directly with the transport of energy through a medium containing particles, and it is based on the differential equation of radiative transfer. Multiple scattering theory is rigorous, it starts with the wave equation or with Maxwell equations, obtains a solution for the scattering by a single particle, introduces the interaction effects of many particles, and then con- 
TABLE I

PREDICTIONS OF THE MODEL

\begin{tabular}{l|c|c|c}
\hline \hline PARAMETERS & OBSTACLES & Full power density, 2-D & Full power density, 3-D \\
\hline$\gamma \rightarrow 1$ & mostly absorbing & $e^{-\eta r} /(2 \pi r)$ & $e^{-\eta r} /\left(4 \pi r^{2}\right)$ \\
$\gamma \rightarrow 0$ & mostly scattering & $\infty$ & $\sim 1 / r$ \\
$\eta=0$ & no obstacles & $1 /(2 \pi r)$ & $1 /\left(4 \pi r^{2}\right)$ \\
\hline & & Radiated power density, 2-D & Radiated power density, 3-D \\
\hline$\gamma \rightarrow 1$ & mostly absorbing & $e^{-\eta r} /(2 \pi r)$ & $e^{-\eta r} /\left(4 \pi r^{2}\right)$ \\
$\gamma \rightarrow 0$ & mostly scattering & $1 /(2 \pi r)$ & $1 /\left(4 \pi r^{2}\right)$ \\
$\eta=0$ & no obstacles & $1 /(2 \pi r)$ & $1 /\left(4 \pi r^{2}\right)$ \\
\hline \hline
\end{tabular}

siders statistical averages. There are different ways to represent the integral solution to the multiple scattering problem, these include methods based on application of Feynman diagrams [13], [14], [21], Feynman path integrals [11], and Twersky integral equations [21]; these methods have also been applied to describe propagation in the urban and rural environments [4], [25].

Clearly, multiple scattering and transport theories deal with the same phenomena, even though their starting points are different, and there are fundamental relationships between them. These are outlined in the classic book by Ishimaru [21, Chapter 14], which shows that under certain approximations it is possible to derive the transport equation using analytical theory. We now relate our proposed wandering photon model to transport theory and then also to multiple scattering theory due to their tight connection.

The (numerical) solution of the radiative transfer equation in the case of a spherical wave radiating in a random medium of isotropic scatterers is outlined in Ishimaru [21, Chapter 12]. In some limiting cases, analytic results are known and are in agreement with the results of our model depicted in Table I. In addition, our random walks model can provide an interesting mathematical interpretation of the diffusive regime. As we move toward a lossless medium $(\gamma \rightarrow 0)$ the radiated power density in 3-D tends to $1 /\left(4 \pi r^{2}\right)$, satisfying the conservation of power, while the full power density has a $1 / r$ dependence. This phenomenon of $1 / r$ slow decay of the full power density is known in transport theory and is due to part of the energy being reflected back toward the source. In this case propagation is purely diffusive [21]. Under the same conditions, in 2-D, the full power density diverges with a log singularity. Our interpretation is in terms of recurrence properties of random walks. Random walks in 2-D are recurrent, hence, when there is no absorption, the same photon visits infinitely many times the same disc of arbitrary radius $\epsilon$. On the contrary, in 3-D the probability of the random walk to revisit the same sphere of arbitrary radius $\epsilon$ is less than one, hence, perfectly reflecting scatterers lead only to a decrease of the rate of decay of the full power density from $1 / r^{2}$ to $1 / r$, due to a finite number of recurrent visits and eventual diffusion of photons in space.

Note also that if particles are mostly absorbing, the diffusion effect becomes negligible and attenuation in 3-D becomes of the type $e^{-\eta r} /\left(4 \pi r^{2}\right)$.

A complete comparison with transport theory is given in Fig. 2, where we reproduce the plots numerically obtained in [21] for $4 \pi r^{2} S(r)$ (continuous line curves), and $4 \pi r^{2} P(r)$ (dashed line curves), and compare them with the corresponding plots of our analytic solution. Note that the parameters of the two theories are different: we use the absorption coefficient $(\gamma)$ and the average step length $(1 / \eta)$ of the random walk, whereas transport theory uses the particles number per unit volume $(\rho)$ and the absorption $\left(\sigma_{a}\right)$, scattering $\left(\sigma_{s}\right)$, and total $\left(\sigma_{t}=\sigma_{a}+\sigma_{s}\right)$ particles cross section. Accordingly, the transport theory curves are parametrized by the albedo $W_{0}=\sigma_{s} / \sigma_{t}$, that is not independent from the abscissa coordinate $\rho \sigma_{t} r$; whereas the wandering photon curves are parametrized by the absorption coefficient $\gamma$, that is totally independent from the abscissa coordinate $\eta r$. In any case, similarity between the numerical solution of [21] and our analytic one is evident.

\section{THE RADIO LINK EQUATION}

We want to validate our theoretical model with experimental data. In order to fit our results to measured data we need to introduce a radio link equation, converting the densities expressed by (11) and (12) to the actual received power of the antenna in our experiments. We do so by adding a multiplicative factor $C$ to (11) and (12) that accounts for the transmitted power, gain of the transmitting antenna, effective area of the receiving antenna, and additional hardware losses in the system. Accordingly, we obtain the following radio link equation for the power received by an antenna located at a distance $r$ from the transmitter

$$
P_{\text {recv }}(r)=\frac{C}{r^{2}} f(\eta r, \gamma)
$$

where $f(\cdot)$, which is $r^{2}$ times either (11) or (12), is a function of the two dimensionless parameters $\eta r$ and $\gamma$, and $C$ is expressed in $\left[\mathrm{Wm}^{2}\right]$ units. Note that usage of (11) or (12) in the expression for the power received, corresponds to two different ideal measurements: one measures the flux of photons passing through an antenna of given area oriented toward the source; the other measures the total number of photons passing through the antenna, coming from any direction. Hence, (11) is more suited to model directional receiving antennas, while (12) is more suited to model isotropic receiving antennas. In practice, when we tune the parameters of both formulas to experimental data, we find that the same result is observed in both cases, of a propagation loss that, due to absorption, presents a smooth transition from free space propagation to exponential attenuation (see Fig. 3). This effect can be seen from (11) and (12), that reduce to $1 /\left(4 \pi r^{2}\right)$ in the near field $(\eta r \ll 1)$, and to a term proportional to $e^{-\left[1-(1-\gamma)^{2}\right] \eta r} / r^{2}$ in the far field $(\eta r \gg 1)$. This 

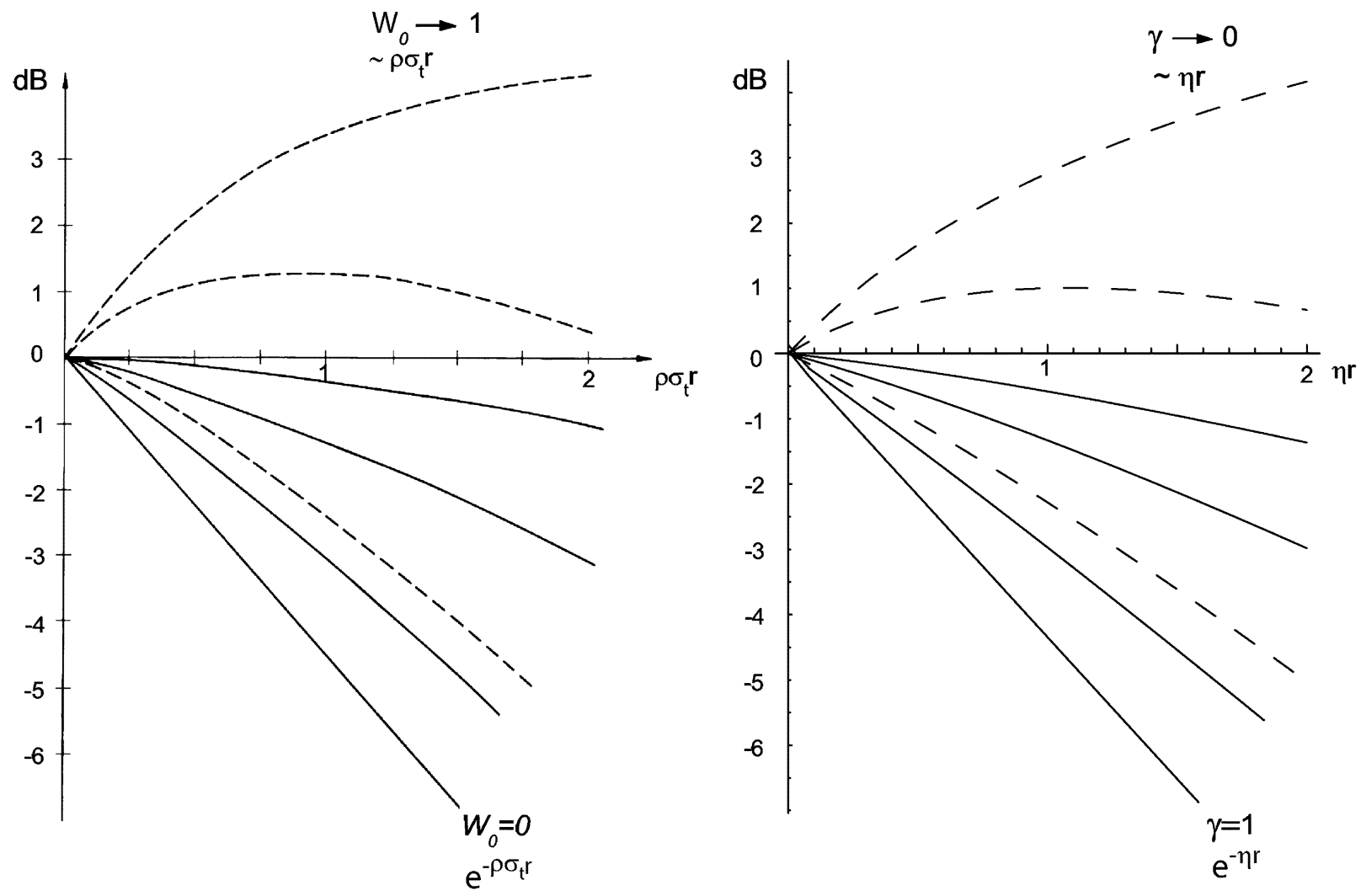

Fig. 2. Power Flux and Power Density. The dashed curves represent the full power density (normalized by a $4 \pi r^{2}$ factor), and the solid line curves represent the radiated power density (normalized by an $4 \pi r^{2}$ factor). Left-hand side: results from transport theory obtained numerically solving the equation of transfer. Right-hand side: analytical results according to the wandering photon model. Curves are parametrized by different absorption values. Perfectly absorbing obstacles lead to an exponential over $r^{2}$ type attenuation. Perfectly reflecting obstacles lead to a $1 / r$ diffusive regime for the full power density and to a $1 /\left(4 \pi r^{2}\right)$ regime for the radiated power density.
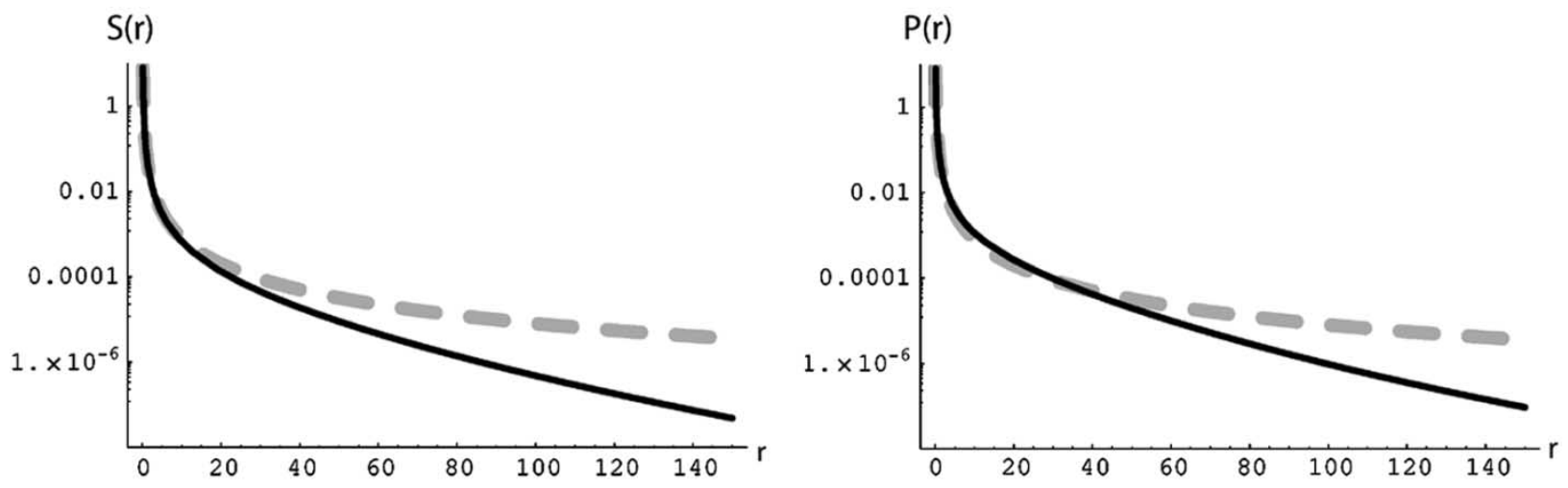

Fig. 3. Results of the model compared to free space propagation. The dashed line curves represent the free space $1 /\left(4 \pi r^{2}\right)$ attenuation law, the continuous line curves represent the predicted radiated power density $S(r)$ (left handside of the figure) and full power density $P(r)$ (right handside of the figure) of our 3-D probabilistic model. We note that the radiated power density model presents a smooth transition from the $1 /\left(4 \pi r^{2}\right)$ law to an exponential attenuation; the full power density model, although providing a very similar fit, predicts higher power near the source due to reflections from obstacles. The breakpoint at which the model starts diverging from the $1 / r^{2}$ law $(r \approx 30 \mathrm{~m}$ in the figure) is determined by the values of $\eta$ and $\gamma$. In the figure these values are determined by fitting experimental data.

suggests using a simplified formula, that can be used for quick, practical prediction purposes, of the type

$$
P_{\text {recv }}(r)=B \frac{e^{-b r}}{r^{2}}
$$

The above formula proves to be as powerful in fitting experimental data as the complete formulas derived directly from the theoretical model. In Fig. 4, we visually compare results of the simplified formula with the formula for the radiated power density. Values of the rms errors are practically indistinguishable at $\sigma_{\text {std }}=3.65 \mathrm{~dB}$ for the radiated power density, $\sigma_{\mathrm{std}}=3.57 \mathrm{~dB}$ for the full power density and $\sigma_{\text {std }}=3.75 \mathrm{~dB}$ for the simplified formula. The main advantage of the exponential formula in (14) is that it can be easily used for calculations by communication engineers. As a matter of fact, it has been successfully assumed in [31] to derive new bounds on the capacity of wireless ad hoc networks. 


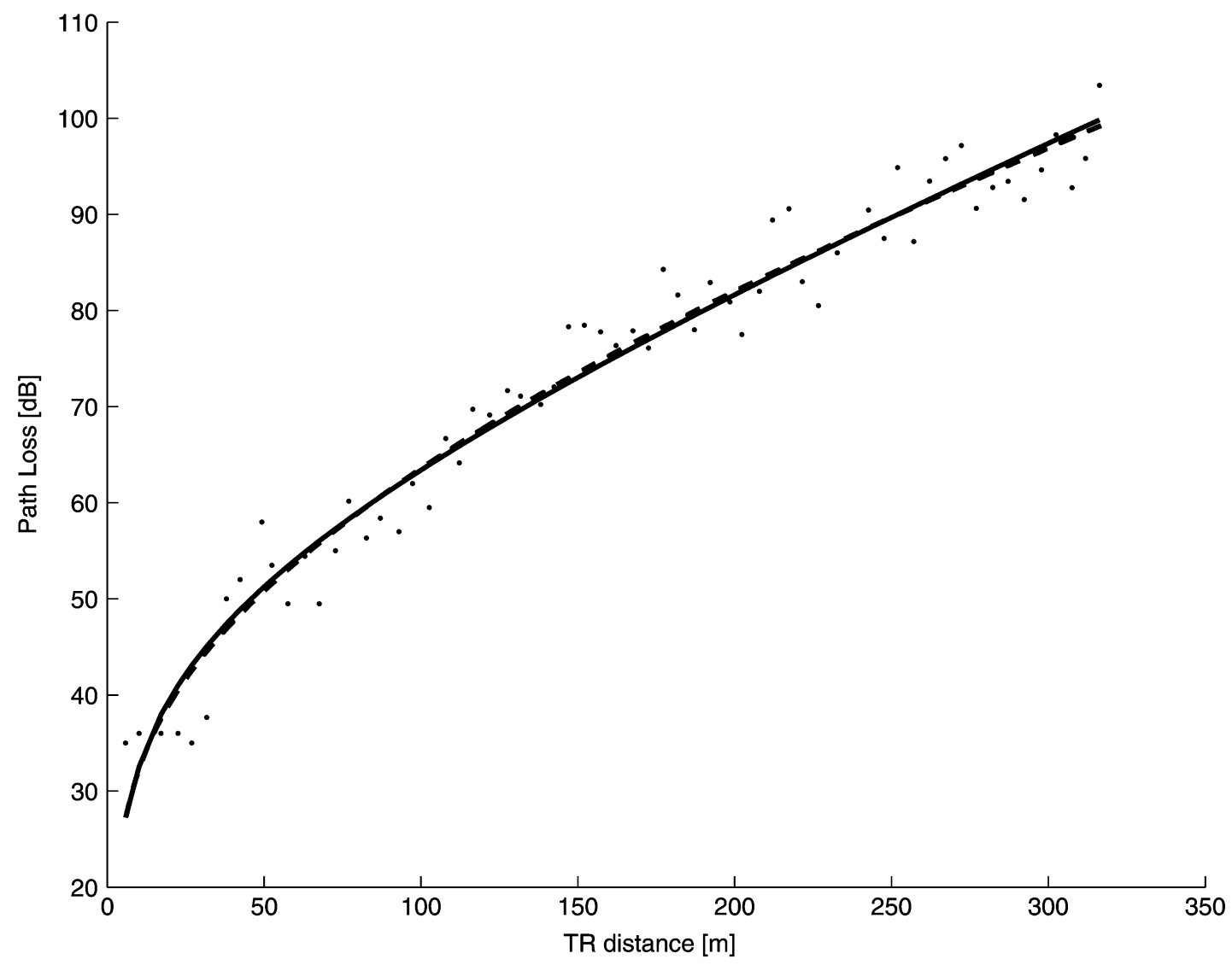

Fig. 4. Complete versus simplified formula. The scattered points represent measured data. The dashed line represent the fit of the data using our radiated power density model. The continuous line represents the fit of the data using our simplified formula (14). The rms error of the radiated power density model, is $\sigma_{\mathrm{std}}=3.65 \mathrm{~dB}$; the rms error of the simplified formula is $\sigma_{\mathrm{std}}=3.75 \mathrm{~dB}$.

\section{EXPERIMENTAL SETUP}

Experimental measurements, collected in Rome, Italy, are courtesy of Ericsson Telecomunicazioni SpA. The transmitting antenna was a vertical dipole located at a height of $6 \mathrm{~m}$ and transmitting a continuous wave $(\mathrm{CW})$ at a frequency of $900 \mathrm{MHz}$, with a radiated power $P_{t}=6.3 \mathrm{~W}$, transmitting antenna gain $G_{t}=2 \mathrm{~dB}$, and EIRP $=P_{t} G_{t}=40 \mathrm{dBm}$. The antenna was located in "Piazza dei Quiriti," in the "Prati" district of Rome, near the Vatican city, at 12.465 91E latitute, $41.91017 \mathrm{~N}$ longitude (see map in Fig. 5). The receiving antenna, a dipole of $G_{r}=2 \mathrm{~dB}$, was mounted on top of a moving vehicle at a height of approximately $1.5 \mathrm{~m}$. The vehicle was equipped with a GPS, to record the position of each sampled data point, and drove around the transmitting site (see right handside of Fig. 5). Each data point consisted of the received power averaged over 50 measurements along a path of 40 wavelengths (Lee method).

Fig. 6 depicts the collected data: the received power is represented by a vertical line drawn at each data point. Fig. 7 depicts a view of the location where some of the measurements were taken and shows the presence of different scatterers in the environment. For subsequent analysis, a reduction in the size of the data was performed as follows. The receiving equipment rounded the smallest value of received power to $-114 \mathrm{~dB}$; therefore, in order to have an un-biased set of data, we considered only points located at a radial distance from the transmitter less than $317 \mathrm{~m}$, for which the received power was always higher than $-114 \mathrm{~dB}$. Moreover, the vehicle moving around the transmitting site collected a larger amount of data points as the radial distance from the transmitter increased. For example, a larger number of data points were collected on an ideal circle of radius $200 \mathrm{~m}$ centered at the transmitting site, compared to the number of data points collected on an ideal circle of radius $50 \mathrm{~m}$. Hence, in order to remove this unwanted bias toward farther points, we averaged all data points inside annuli of $5 \mathrm{~m}$ intervals, generating a uniform set of measurements along the radial direction. This corresponds to performing a local spatial average similar to the standard Lee method, but performed on a small annular area.

\section{A. Path Loss}

We use (13) to define the measured Path Loss for the considered experiments as the expression in $\mathrm{dB}$ :

$$
\mathrm{PL}(r)=-\left[P_{\text {recv }}(r)\right]_{d B}
$$

and the rms error of the predicted path loss from the sampled data as

$$
\sigma_{\text {std }}=\sqrt{\frac{\sum_{j=1}^{n}\left[\mathrm{PL}_{m}\left(r_{j}\right)-\mathrm{PL}\left(r_{j}\right)\right]^{2}}{n}}
$$

where $n$ is the number of measured data points, $\mathrm{PL}_{m}\left(r_{j}\right)$ is the measured path loss at the jth point located at a radial dis- 

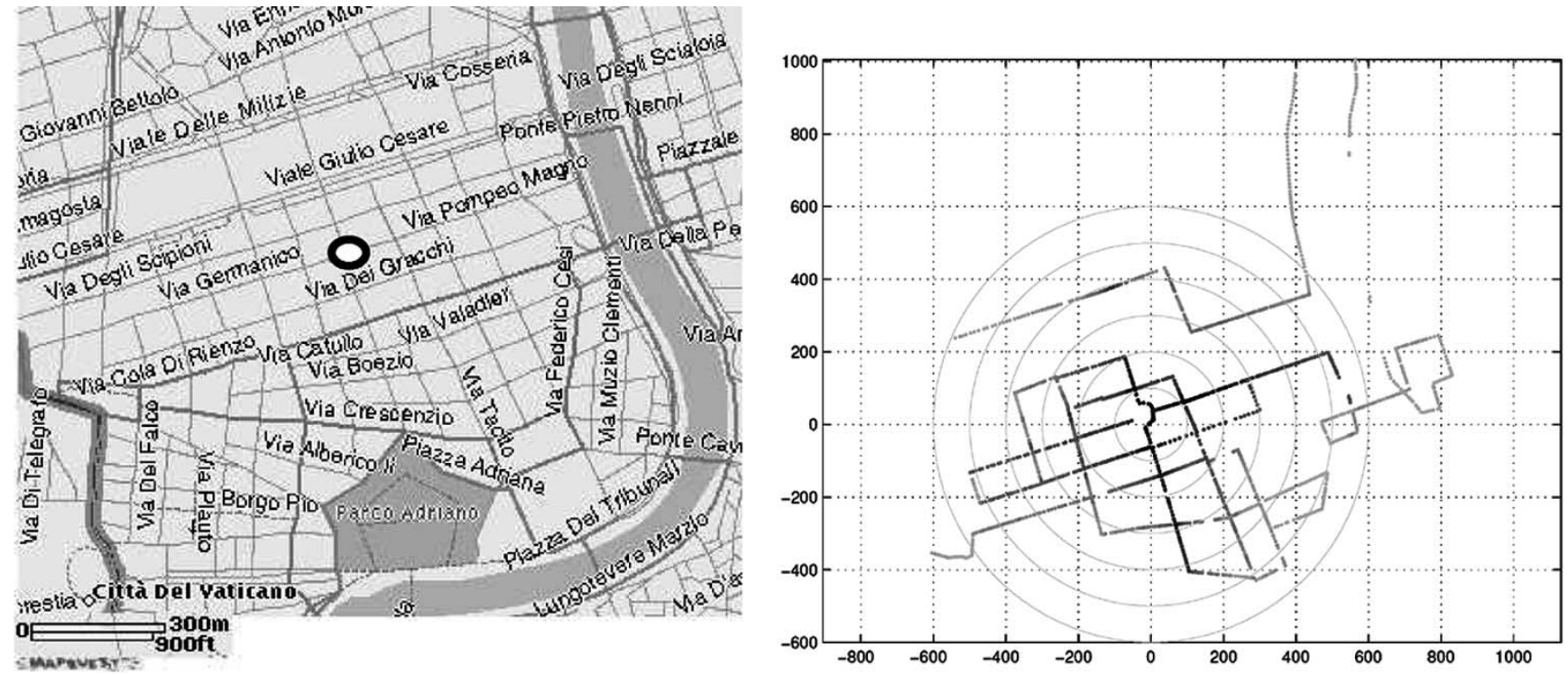

Fig. 5. Measurements location. Measurements were taken in the "Prati" district of Rome, near the Vatican city. The transmitting antenna was placed in "Piazza dei Quiriti," which is highlighted by the black circle in the map on the left handside of the figure. The right-hand side of the figure shows the path taken by the van as it drove around the transmitting site. Each data point is shown with a gray level proportional to the power of the received signal. Circles centered at the transmitting site are drawn with increasing radii of $100 \mathrm{~m}$ steps. The map on the left hand side of the figure is taken from http://www.mapquest.com. Power level measurements are courtesy of Ericsson Telecomunicazioni SpA.

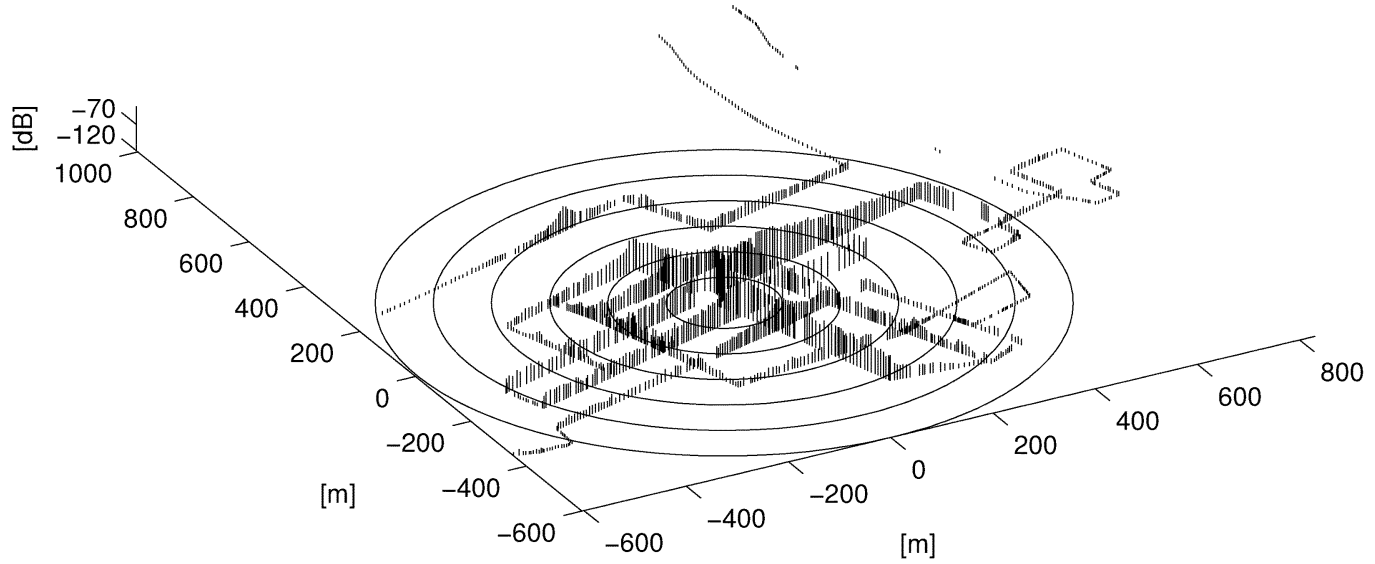

Fig. 6. Plot of the received power. A vertical line is drawn at each data point. The height of the line corresponds to the value of the received power expressed in $\mathrm{dB}$. Circles centered at the transmitting site are drawn with increasing radii of $100 \mathrm{~m}$ steps.
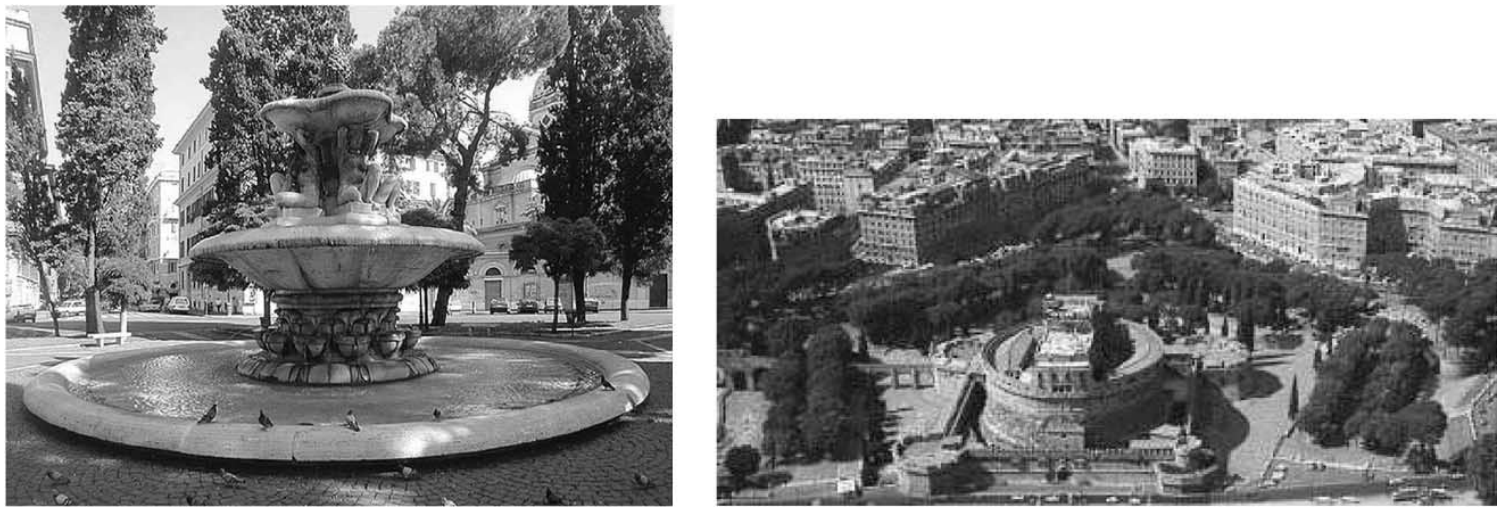

Fig. 7. View of the location. The left handside of the figure depicts "Piazza dei Quiriti," where the transmitting antenna was located. The right handside of the picture depicts a view of "Parco Adriano" and of the surrounding streets where some of the measurements were taken.

tance $r_{j}$ from the transmitter, and $\mathrm{PL}\left(r_{j}\right)$ is the corresponding predicted path loss derived from (15). To minimize the rms error we use the standard MATLAB procedure lsqnonlin( ) that is a fairly sophisticated non linear least squares routine 


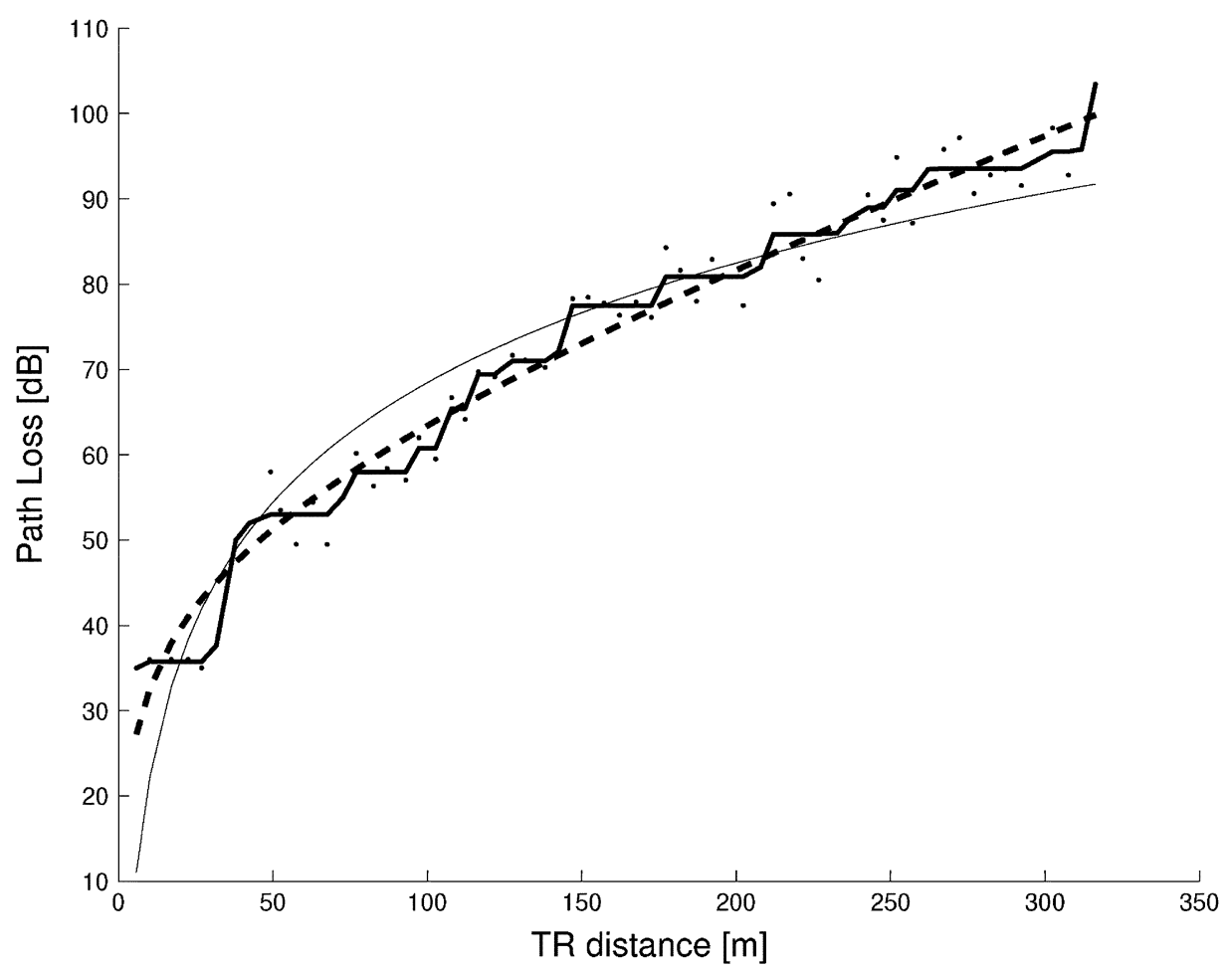

Fig. 8. Comparison. The dashed line represent the fit of the data using the simplified path loss formula. The thin continuous line represents the fit of the data using a power law. The "staircase" line is the result of the monotonic regression, representing the best nondecreasing function fitting the data. Values of the rms error are reported in Table II.

based on the Gauss-Newton algorithm. Accordingly, we find the values $\eta=0.10, \gamma=0.14, C=0.073$, and a corresponding $\sigma_{\mathrm{std}}=3.65 \mathrm{~dB}$, for the radiated power density model; and the values $\eta=0.13, \gamma=0.10, C=0.037, \sigma_{\text {std }}=3.57 \mathrm{~dB}$, for the full power density model. These values correspond to having on average one obstacle every 10-13 m, with an absorption coefficient of $10 \%-14 \%$, which is very reasonable for the considered urban area. In addition, we would expect the factor $C$ for the radiated power density to be equal to the transmitted power times the transmitting antenna gain and the effective area $A$ of the receiving antenna. Accordingly, we get $C=(\mathrm{EIRP}) A=0.069$ for the experimental equipment and radiated power, where we have included the extra factor 0.5 accounting for the random incident polarization on the receiving dipole.

\section{B. Comparison With Other Formulas and Best Fit}

In Fig. 8 we compare our results with the simple power law model (Hata formula [19]) and with the result of monotonic regression performed on the measured data. The monotonic regression [1] of a function is the best, in mean square, nondecreasing function fitting the data. It provides a lower bound on the minimum value of $\sigma_{\text {std }}$ that can be achieved by any theoretical model of propagation: any path loss prediction that does not depend on specific knowledge of an inhomogeneous environment, and in particular that is a function only of distance from the transmitter and general properties of the environment, must be a nondecreasing function of distance. Results are summed up in Table II, and validate our model that closely follows the monotonic regression line depicted in Fig. 8.
TABLE II

REgRESSION RESUlts. THE FiRST LiNE OF THE TABLE Is RELATIVE TO THE Hata Formula. The Second Line Is Relative to the SimPlified FORMULA OF (14). THE THIRD LINE IS RELATIVE TO MONOTONIC REGRESSION

\begin{tabular}{l|c|c|c}
\hline Model & $\sigma_{\text {std }}$ & Exponent & Multiplicative Factor \\
\hline & & & \\
$P L^{-1}(r)=A\left(\frac{1}{r}\right)^{a}$ & $6.05 d B$ & $a=4.66$ & $A=.0034$ \\
$P L^{-1}(r)=B \frac{e^{-b r}}{r^{2}}$ & $3.75 d B$ & $b=0.028$ & $B=.0765$ \\
Monotonic Regression & $2.04 d B$ & & \\
\hline
\end{tabular}

From Fig. 8 it is also evident what is the typical problem of applying the simple Hata formula, popular in cellular systems design, to predict the path loss in microcellular systems: a power law with a fixed exponent (4.66 in this case) overestimates the real path loss in the close range, where the signal undergoes few reflections to reach the receiver and propagation is almost as in free space, and it underestimates the real path loss at larger distances, where there is an exponential attenuation, due to the absorption of many reflecting obstacles. Empirical formulas characterized by two different power laws, one with an exponent close to two, to be used close to the transmitter, and another with a high order exponent, to be used after a breakpoint distance, are better suited for prediction in this kind of environments and can be theoretically justified by our model.

Comparison with other theoretical expressions is also desirable. This is not easy, because available formulas usually rely on a large number of parameters and a physically sound interpolation becomes difficult. We compared our results with those of Blaunstein and Bach Andersen [4], who provide path loss theoretical expressions for the three cases of rural, mixed, and 

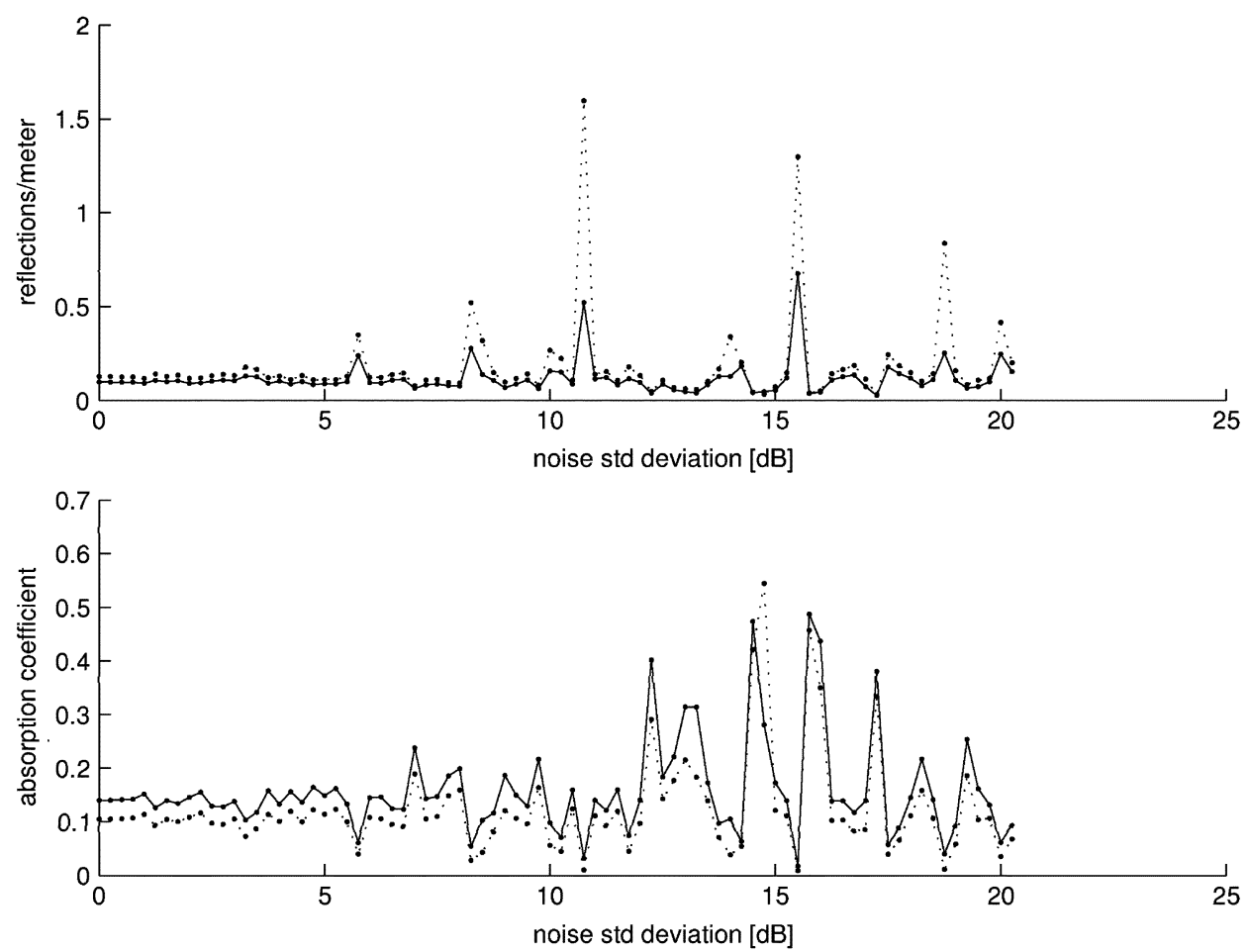

Fig. 9. Sensitivity analysis. Predictions of the radiated power density model are indicated with continuous lines, predictions of the full density model are indicated with dotted lines.

urban areas. It turned out that their best fit with our experiments was obtained for the rural model, with a mean square error, $\sigma_{\text {std }}=4.61 \mathrm{~dB}$, larger than ours. The other two models provided deviations larger than $6 \mathrm{~dB}$, and best fit interpolated parameters where not always sound on physical bases. These results lead us to conclude that propagation in towns illuminated by low antennas is dominated by the disseminated large amount of relatively small and diffusing obstacles that lead to exponential attenuation in the far range, that is not captured by the urban and suburban model of [4].

\section{Sensitivity to Random Noise}

As indication of robustness of our theoretical model, we check if the physical parameters, absorption coefficient $(\gamma)$ and average number of reflections per meter $(\eta)$, are stable under random perturbation of the data. We do so by adding log-normal random noise of zero mean and standard deviation $\sigma_{\text {noise }}$ to the data and determining, via non linear regression, the parameters $\eta$ and $\gamma$ for different values of $\sigma_{\text {noise }}$. Results are presented in Fig. 9. We note that the parameters are very stable under perturbations up to $10 \mathrm{~dB}$. This suggests that they capture real physical properties of the environment that are invariant to random noise.

\section{CONCLUSION}

This paper presented a simple model of propagation in microcells based on only two physical parameters: the amount of clutter and the amount of absorption in the environment. The model is entirely based on probability theory, rather than on the classical theory of electromagnetics. We have solved the model analytically and derived a power attenuation formula that has been validated with experimental data. Despite its crude simplifications, the model correctly predicts a path loss that follows (in 3-D) an inverse square law with the distance to the transmitter, with a smooth transition to an exponential mode, as the distance between transmitter and receiver increases. This result gives a theoretical basis for understanding the qualitative phenomenon that was previously described by empirically derived double exponent models (models with a transition from low to high order power laws as a function of distance from the transmitter), while providing a simpler mathematical model.

We believe that the probabilistic approach exploited here is a powerful tool to describe the complex urban wireless channel. Future research directions include characterization of wide band channels and extension of the study from average quantities to their second order moments.

\section{APPENDIX A \\ CAlCUlation OF $G(r), 2-\mathrm{D}$}

We start by computing the 2-D FT of $Q(r)$ :

$$
q(u, v)=\int_{-\infty}^{+\infty} \int_{-\infty}^{+\infty} \frac{\eta e^{-\eta \sqrt{x^{2}+y^{2}}}}{2 \pi \sqrt{x^{2}+y^{2}}} e^{-i u x} e^{-i v y} d x d y
$$

By letting $x=r \cos \phi, y=r \sin \phi, u=\omega \cos \psi, v=\omega \sin \psi$, we have

$$
\begin{aligned}
q(\omega) & =\frac{\eta}{2 \pi} \int_{0}^{\infty} e^{-\eta r} d r \int_{0}^{2 \pi} e^{-i \omega r \cos (\phi-\psi)} d \phi \\
& =\frac{\eta}{2 \pi} \int_{0}^{\infty} e^{-\eta r} d r \int_{0}^{2 \pi} \sum_{k=-\infty}^{+\infty}(-i)^{k} J_{k}(\omega r) e^{-i k(\phi-\psi)} d \phi \\
& =\eta \int_{0}^{\infty} e^{-\eta r} J_{0}(\omega r) d r
\end{aligned}
$$


where we use the Bessel functions $J_{k}(\cdot)$, and exploited the Bessel expansion 8.511 .4 in [15]. Then, by means of identity 6.611 .1 of [15], we obtain

$$
q(\omega)=\frac{\eta}{\sqrt{\eta^{2}+\omega^{2}}} .
$$

Substituting $q(\omega)$ into (2), we obtain

$$
g(\omega)=\frac{\gamma \eta}{\sqrt{\eta^{2}+\omega^{2}}-(1-\gamma) \eta}
$$

and by inverse FT we obtain

$$
\begin{aligned}
G(r)= & \frac{\gamma \eta}{(2 \pi)^{2}} \int_{0}^{\infty} \frac{\omega}{\sqrt{\eta^{2}+\omega^{2}}-(1-\gamma) \eta} d \omega \\
& \times \int_{0}^{2 \pi} e^{i \omega r \cos (\psi-\phi)} d \psi \\
= & \frac{\gamma \eta}{(2 \pi)^{2}} \int_{0}^{\infty} \frac{\omega}{\sqrt{\eta^{2}+\omega^{2}}-(1-\gamma) \eta} d \omega \\
& \times \int_{0}^{2 \pi} \sum_{k=-\infty}^{+\infty} i^{k} J_{k}(\omega r) e^{i k(\psi-\phi)} d \psi \\
= & \frac{\gamma \eta}{2 \pi} \int_{0}^{\infty} \frac{\omega J_{0}(\omega r)}{\sqrt{\omega^{2}+\eta^{2}}-(1-\gamma) \eta} d \omega .
\end{aligned}
$$

Note that, for $\gamma \rightarrow 1$ (perfectly absorbing obstacles), the photon is absorbed at the first step of the random walk, and the integral reduces to

$$
\frac{\eta}{2 \pi} \int_{0}^{\infty} \frac{\omega J_{0}(\omega r)}{\sqrt{\omega^{2}+\eta^{2}}} d \omega=\frac{\eta e^{-\eta r}}{2 \pi r}=Q(r)
$$

as expected, see identity 6.554.1 in [15].

In the general case of $\gamma \neq 1$ the integral in (16) is nontrivial to compute. An exact solution, expressed in terms of an infinite series of Bessel polynomials, is derived in the following section. A closed form approximation is shown in Appendix 1-B.

\section{A. Exact Expression in Terms of a Series}

In this section we show the exact derivation of $G(r)$ in 2D, written as a series of Bessel polynomials. Let $\alpha=\eta(1-\gamma)$. We rewrite (16) as follows:

$$
\begin{aligned}
G(r)= & \frac{\gamma \eta}{2 \pi} \int_{0}^{\infty} \frac{\omega J_{0}(\omega r)}{\sqrt{\omega^{2}+\eta^{2}}-\alpha} d \omega \\
= & \frac{\gamma \eta}{2 \pi} \int_{0}^{\infty} \frac{\omega J_{0}(\omega r)}{\omega^{2}+\eta^{2}-\alpha^{2}}\left(\sqrt{\omega^{2}+\eta^{2}}+\alpha\right) d \omega \\
= & \frac{\gamma \eta}{2 \pi}\left[\alpha \int_{0}^{\infty} \frac{\omega J_{0}(\omega r)}{\omega^{2}+\eta^{2}-\alpha^{2}} d \omega\right. \\
& \left.+\int_{0}^{\infty} \frac{\omega J_{0}(\omega r) \sqrt{\omega^{2}+\eta^{2}}}{\omega^{2}+\eta^{2}-\alpha^{2}} d \omega\right] \\
= & \frac{\gamma \eta}{2 \pi}\left(\widehat{\mathcal{I}_{1}}+\widehat{\mathcal{I}}_{2}\right)=\frac{\gamma \eta}{2 \pi}\left[\alpha K_{0}\left(\sqrt{\eta^{2}-\alpha^{2}} r\right)+\widehat{\mathcal{I}}_{2}\right]
\end{aligned}
$$

having used identity 6.532.4, in [15], to calculate integral $\widehat{\mathcal{I}}_{1}$. We can then write integral $\widehat{\mathcal{I}}_{2}$ as a series of integrals

$$
\begin{aligned}
\widehat{\mathcal{I}_{2}} & =\int_{0}^{\infty} \frac{\omega J_{0}(\omega r)}{\sqrt{\omega^{2}+\eta^{2}}\left(1-\frac{\alpha^{2}}{\omega^{2}+\eta^{2}}\right)} d \omega \\
& =\int_{0}^{\infty} \frac{\omega J_{0}(\omega r)}{\sqrt{\omega^{2}+\eta^{2}}} \sum_{n=0}^{\infty} \frac{\alpha^{2 n}}{\left(\omega^{2}+\eta^{2}\right)^{n}} d \omega \\
& =\sum_{n=0}^{\infty} \alpha^{2 n} \int_{0}^{\infty} \frac{\omega J_{0}(\omega r)}{\left(\omega^{2}+\eta^{2}\right)^{n+1 / 2}} d \omega=\sum_{n=0}^{\infty} \mathcal{I}_{n} .
\end{aligned}
$$

We have the following recurrence relation:

$$
\left\{\begin{array}{l}
\mathcal{I}_{0}=\int_{0}^{\infty} \frac{\omega J_{0}(\omega r)}{\sqrt{\omega^{2}+\eta^{2}}} d \omega=\frac{e^{-\eta r}}{r} \\
\mathcal{I}_{n+1}=\frac{-\alpha^{2}}{(2 n+1)} \frac{1}{\eta} \frac{\partial}{\partial \eta} \mathcal{I}_{n} .
\end{array}\right.
$$

We recall that Bessel polynomials [18] are defined as:

$$
\theta_{n}(z)=\sum_{j=0}^{n} a_{j} z^{n-j}
$$

where all coefficients are integers given by

$$
a_{j}=\frac{(n+j) !}{2^{j}(n-j) ! j !} .
$$

From the recurrence (19) and the definition of $\theta_{n}$, it follows that:

$$
\widehat{\mathcal{I}_{2}}=\sum_{n=0}^{\infty} \mathcal{I}_{n}=\frac{e^{-\eta r}}{r}\left[1+\eta r \sum_{n=0}^{\infty} c_{n} \theta_{n}(\eta r)\right]
$$

where

$$
c_{n}=\frac{(1-\gamma)^{2(n+1)}}{(2 n+1) ! !}
$$

which, after substitution in (17), gives the following exact formula:

$$
\begin{aligned}
G(r)=\frac{\gamma \eta}{2 \pi}\left[\frac{e^{-\eta r}}{r}\left(1+\eta r \sum_{n=0}^{\infty} c_{n} \theta_{n}(\eta r)\right)\right. & \\
& \left.+\alpha K_{0}\left(\sqrt{\eta^{2}-\alpha^{2}} r\right)\right]
\end{aligned}
$$

\section{B. Approximate Expression}

In order to obtain a closed-form formula for $G(r)$, we approximate the series that appears in (18). We consider the Taylor expansion of the following function of $\eta$ :

$\frac{1}{\sqrt{\omega^{2}+\left(\eta-\frac{\alpha^{2}}{\eta}\right)^{2}}}=\frac{1}{\sqrt{\omega^{2}+\eta^{2}}}+\frac{1}{2} \frac{2 \eta}{\left(\omega^{2}+\eta^{2}\right)^{3 / 2}} \frac{\alpha^{2}}{\eta}+\cdots$ 
and note that the first two terms of this series coincide with the first two terms of the series in (18). Hence, neglecting higher order terms, we write

$$
\widehat{\mathcal{I}_{2}} \approx \int_{0}^{\infty} \frac{\omega J_{0}(\omega r)}{\sqrt{\omega^{2}+\left(\eta-\frac{\alpha^{2}}{\eta}\right)^{2}}} d \omega=\frac{e^{-\frac{\eta^{2}-\alpha^{2}}{\eta} r}}{r}
$$

where we have recognized the integral in (23) as the inverse FT of $q(\omega)$, with parameter $\eta-\alpha^{2} / \eta$. Finally, substituting (23) into (17), we obtain the desired approximation

$$
G(r) \approx \frac{\gamma \eta}{2 \pi}\left[\frac{e^{-\frac{\eta^{2}-\alpha^{2}}{\eta} r}}{r}+\alpha K_{0}\left(\sqrt{\eta^{2}-\alpha^{2}} r\right)\right] .
$$

Note that, in spite of being an approximation, (24) is a probability density; we see here that it integrates to one

$$
\begin{aligned}
& \int_{0}^{2 \pi} d \phi \int_{0}^{\infty} r \frac{\gamma \eta}{2 \pi}\left[\frac{e^{-\frac{\eta^{2}-\alpha^{2}}{\eta} r}}{r}+\alpha K_{0}\left(\sqrt{\eta^{2}-\alpha^{2}} r\right)\right] d r \\
& \quad=\gamma \eta\left(\frac{\eta}{\eta^{2}-\alpha^{2}}+\frac{\alpha}{\eta^{2}-\alpha^{2}}\right) \\
& \quad=\frac{\gamma \eta}{\eta-\alpha}=1 .
\end{aligned}
$$

We now discuss the validity of the approximation, numerically estimating the series of Bessel polynomials in (20), and comparing it with (23). Letting $\eta r=R$, we have

$$
\begin{aligned}
\widehat{\mathcal{I}}_{2} & =\eta\left[\frac{e^{-R}}{R}\left(1+R \sum_{n=0}^{\infty} c_{n} \theta_{n}(R)\right)\right] \\
& \approx \operatorname{Appr}\left(\widehat{\mathcal{I}}_{2}\right)=\eta\left[\frac{e^{-R}}{R} e^{(1-\gamma)^{2} R}\right] .
\end{aligned}
$$

Note that it would be misleading to refer to the relative error, because both $\widehat{\mathcal{I}_{2}}$ and $\operatorname{Appr}\left(\widehat{\mathcal{I}_{2}}\right)$ tend to zero, as $R \rightarrow \infty$. Accordingly, we evaluate the absolute error. Letting Err $=\widehat{\mathcal{I}_{2}}-$ $\operatorname{Appr}\left(\widehat{\mathcal{I}}_{2}\right)$, we depict in Fig. 10 a plot of the function:

$$
\left(\frac{\text { Err }}{\eta}\right)^{2}=\left\{\frac{e^{-R}}{R}\left[1+R \sum_{n=0}^{\infty} c_{n} \theta_{n}(R)-e^{(1-\gamma)^{2} R}\right]\right\}^{2}
$$

numerical estimation suggests that the error is bounded by:

$$
|\operatorname{Err}|<\sqrt{0.0027} \eta
$$

\section{APPENDIX B}

\section{CALCUlation of $G(r), 3-\mathrm{D}$}

We start by computing the 3-D FT of $Q(r)$ :

$$
\begin{aligned}
q(\omega) & =\int_{0}^{2 \pi} d \phi \int_{0}^{\pi} \sin \theta d \theta \int_{0}^{\infty} r^{2} e^{-i \omega \cos \theta r} Q(r) d r \\
& =\frac{\eta}{2} \int_{0}^{\pi} \sin \theta d \theta \int_{0}^{\infty} e^{-(\eta+i \omega \cos \theta) r} d r \\
& =\frac{\eta}{2} \int_{0}^{\pi} \frac{\sin \theta}{\eta+i \omega \cos \theta} d \theta=\frac{\eta}{\omega} \arctan \frac{\omega}{\eta} .
\end{aligned}
$$

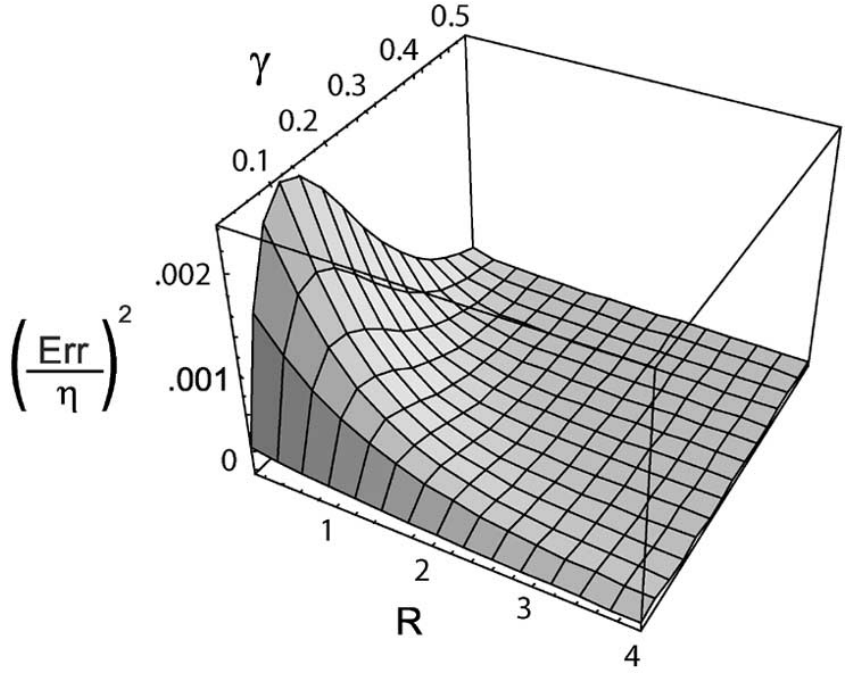

Fig. 10. Estimate of the Error. The plot depicts an estimate of the squared error of the approximation.

Substituting $q(\omega)$ into (2), we obtain

$$
g(\omega)=\frac{\gamma \frac{\eta}{\omega} \arctan \frac{\omega}{\eta}}{1-(1-\gamma) \frac{\eta}{\omega} \arctan \frac{\omega}{\eta}}
$$

by inverse FT we have

$$
\begin{aligned}
G(r) & =\frac{1}{(2 \pi)^{3}} \int_{0}^{2 \pi} d \phi \int_{0}^{\pi} \sin \theta d \theta \int_{0}^{\infty} \omega^{2} g(\omega) e^{i \omega r \cos \theta} d \omega \\
& =\frac{1}{(2 \pi)^{2}} \int_{0}^{\infty} \omega^{2} g(\omega) d \omega \int_{0}^{\pi} \sin \theta e^{i \omega r \cos \theta} d \theta \\
& =\frac{2}{(2 \pi)^{2}} \int_{0}^{\infty} \omega^{2} g(\omega) \operatorname{sinc}(\omega r) d \omega
\end{aligned}
$$

where we have defined $\operatorname{sinc} x=(\sin x) / x$.

We now introduce the following approximation (see [15], 1.644.1)

$$
\arctan \frac{\omega}{\eta} \approx \frac{\omega / \text { eta }}{\sqrt{1+\left(\frac{\omega}{\eta}\right)^{2}}} .
$$

Substituting (25) into (26) we obtain

$$
\begin{aligned}
G(r) \approx & \frac{2}{(2 \pi)^{2}} \int_{0}^{\infty} \omega^{2} \frac{\gamma \eta}{\sqrt{\eta^{2}+\omega^{2}}-(1-\gamma) \eta} \operatorname{sinc}(\omega r) d \omega \\
= & \frac{\gamma \eta}{(2 \pi)^{2}}\left[(1-\gamma) \eta \int_{-\infty}^{+\infty} \frac{\omega^{2} \operatorname{sinc}(\omega r)}{\omega^{2}+\eta^{2}-(1-\gamma)^{2} \eta^{2}} d \omega\right. \\
& \left.+\int_{-\infty}^{+\infty} \frac{\omega^{2} \sqrt{\eta^{2}+\omega^{2}} \operatorname{sinc}(\omega r)}{\omega^{2}+\eta^{2}-(1-\gamma)^{2} \eta^{2}} d \omega\right] \\
= & \frac{\gamma \eta}{(2 \pi)^{2}}\left(\widehat{\mathcal{I}_{1}}+\widehat{\mathcal{I}_{2}}\right) \\
= & \frac{\gamma \eta}{(2 \pi)^{2}}\left[(1-\gamma) \eta \frac{\pi}{r} e^{-\sqrt{1-(1-\gamma)^{2}} \eta r}+\widehat{\mathcal{I}_{2}}\right]
\end{aligned}
$$

having used identities 3.721.1, 3.725.1 in [15] to calculate integral $\hat{\mathcal{I}}_{1}$. 
Letting $\alpha=\eta(1-\gamma)$, we have

$$
\begin{aligned}
\widehat{\mathcal{I}_{2}} & =2 \int_{0}^{+\infty} \frac{\omega^{2} \operatorname{sinc}(\omega r)}{\sqrt{\eta^{2}+\omega^{2}}\left(1-\frac{\alpha^{2}}{\omega^{2}+\eta^{2}}\right)} d \omega \\
& =2 \int_{0}^{+\infty} \frac{\omega^{2} \operatorname{sinc} \omega r}{\sqrt{\omega^{2}+\eta^{2}}} \sum_{n=0}^{\infty} \frac{\alpha^{2 n}}{\left(\omega^{2}+\eta^{2}\right)^{n}} d \omega .
\end{aligned}
$$

We now proceed exactly as in the 2-D case. Exploiting the Taylor expansion in (22), we obtain

$$
\begin{aligned}
& \widehat{\mathcal{I}_{2}} \approx 2 \int_{0}^{+\infty} \frac{\omega^{2} \operatorname{sinc} \omega r}{\sqrt{\omega^{2}+\left(\eta-\frac{\alpha^{2}}{\eta}\right)^{2}}} d \omega \\
&=\frac{2}{\left(\eta-\frac{\alpha^{2}}{\eta}\right)} \int_{0}^{+\infty} \omega^{2} \operatorname{sinc}(\omega r) \frac{\left(\eta-\frac{\alpha^{2}}{\eta}\right)}{\omega} \\
& \times \arctan \left(\frac{\omega}{\eta-\frac{\alpha^{2}}{\eta}}\right) d \omega
\end{aligned}
$$

having used again the approximation in (27). We now recognize the integral above as the inverse FT of $q(\omega)$, with parameter $\eta-\alpha^{2} / \eta$. It follows that

$$
\widehat{\mathcal{I}_{2}} \approx(2 \pi)^{2} \frac{e^{-\left(\eta-\frac{\alpha^{2}}{\eta}\right) r}}{4 \pi r^{2}} .
$$

Substituting (29) into (28), we finally obtain the expression for $G(r)$ given in (9).

As a check, we note that, in spite of being an approximation, (9) integrates to one, and so it is a probability density

$$
\begin{aligned}
& \iiint G(r) d \underline{\mathbf{v}} \\
& =4 \pi \frac{\gamma \eta}{4 \pi}\left\{(1-\gamma) \frac{\eta}{\left[1-(1-\gamma)^{2}\right] \eta^{2}}+\frac{1}{\eta\left[1-(1-\gamma)^{2}\right]}\right\} \\
& =\gamma \frac{2-\gamma}{2 \gamma-\gamma^{2}}=1 .
\end{aligned}
$$

\section{ACKNOWLEDGMENT}

The authors would like to thank Dr. S. De Vita of Ericsson Telecomunicazioni $\mathrm{SpA}$ for providing access to experimental data, and the anonymous reviewers for their comments.

\section{REFERENCES}

[1] R. E. Barlow, D. J. Bartholomew, J. M. Bremner, and H. D. Brunk, Statistical Inference Under Order Restrictions: The Theory and Applications of Isotonic Regression. New York: Wiley, 1972.

[2] H. L. Bertoni, W. Honcharenko, L. R. Maciel, and H. H. Xia, "UHF propagation prediction for wireless personal communications," Proc. IEEE, vol. 82, pp. 1333-1359, Sept. 1994.

[3] N. Blaunstein, "Average field attenuation in the nonregular impedance streed waveguide," IEEE Trans. Antennas Propagat., vol. 64, pp. 1782-1788, Dec. 1998.

[4] N. Blaunstein and J. B. Andersen, Multipath Phenomena in Cellular Networks. Norwood, MA: Artech house, 2002, ch. 4.

[5] N. Blaunstein, R. Giladi, and M. Levin, "Characteristics' prediction in urban and suburban environments," IEEE Trans. Veh. Technol., vol. 47, pp. 225-234, Jan. 1998.
[6] N. Blaunstein, D. Katz, D. Censor, A. Freedman, I. Matiyahu, and I. GurArie, "Prediction of loss characteristics in built-up areas with various buildings' overlay profiles," IEEE Antennas Propagat. Mag., vol. 43, pp. 181-191, 2001.

[7] N. Blaunstein and M. Levin, "Prediction of UHF-wave propagation in suburban and rural environments," in Proc. URSI Symposium on Communication, 1995, pp. 191-200.

[8] - "VHF/UHF wave attenuation in a city with regularly distributed buildings," Radio Science, vol. 31, pp. 313-323, 1996.

[9] V. Erceg, L. Greenstein, S. Tjandra, S. R. Parkoff, A. Gupta, B. Kulic, A. A. Julius, and R. Bianchi, "An empirically based path loss model for wireless channels in suburban environments," IEEE J. Select. Areas Commun., vol. 17, pp. 1205-1211, Aug. 1999.

[10] M. J. Feurstein, K. L. Blackard, T. Rappaport, S. Y. Seidel, and H. H. $\mathrm{Xia}$, "Path loss, delay spread, and outage models as functions of antenna height for microcellular systems design," IEEE Trans. Veh. Technol., vol. 43, no. 3, pp. 487-498, 1994.

[11] R. P. Feynman and A. R. Hibbs, Quantum Mechanics and Path Integrals: McGraw Hill, 1965.

[12] G. Franceschetti, S. Marano, and F. Palmieri, "Propagation without wave equation, toward an urban area model," IEEE Trans. Antennas Propagat., vol. 47, no. 9, pp. 1393-1404, 1999.

[13] V. Frisch, "Wave propagation in random media," in Probabilistic Methods in Applied Mathematics, A. T. B. Reid, Ed. New York: Academic, 1968, vol. 1, pp. 76-198.

[14] N. Markuvitz, "On the theory of plasma turbulence," Journal of Mathematical Physics, vol. 15, pp. 869-879.

[15] I. S. Gradshteyn and I. M. Ryzhik, Table of Integrals, Series, and Products, 5th ed, A. Jeffrey, Ed. New York: Academic, 1994.

[16] E. Green, "Path loss and signal variability analysis for microcells," in Proc. IEE Fifth International Conference on Mobile and Personal Communications, 1989 , pp. 38-41.

[17] L. Greenstein, N. Amitay, T.-S. Chu, L. J. Cimini Jr., G. J. Foschini, M. J. Gans, C.-L. I, A. J. Rustako Jr., R. A. Valenzuela, and G. Vannucci, "Microcells in personal communications systems," IEEE Commun. Mag., pp. 76-88, Dec. 1992.

[18] E. Grosswald, Bessel Polynomials. New York: Springer Verlag, 1978. Lecture Notes in Mathematics 698

[19] M. Hata, "Empirical formula for propagation loss in land mobile radio services," IEEE Trans. Veh. Technol., vol. 29, pp. 317-325, 1980.

[20] B. Hughes, Random Walks and Random Environments, Vol. 1, Random Walks. Oxford, U.K.: Oxford University Press, 1995.

[21] A. Ishimaru, Wave Propagation and Scattering in Random Media. New York: Academic, 1978, vol. 1, 2.

[22] L. R. Maciel, H. L. Bertoni, and H. H. Xia, "Unified approach to prediction of propagation over buildings for all ranges of base station antenna height," IEEE Trans. Veh. Technol., vol. 42, Jan. 1993

[23] S. Marano, F. Palmieri, and G. Franceschetti, "Statistical characterization of ray propagation in a random lattice," J. Optic. Soc. Amer., vol. 16, no. 10, pp. 2459-2464, 1999 .

[24] R. Mazar, A. Bronschtein, and I. T. Lu, "Theoretical analysis of UHF propagation in a city street modelet as a random multislit waveguide," IEEE Trans. Antennas Propagat., vol. 46, pp. 864-871, June 1998.

[25] L. C. Ong, P. Soma, and Y. W. Chia, "Characterization of propagation channel in the presence of an imperfect semi-infinite half-plane using asymptotic path integral and ray tracing techniques," in Proc. 29th European Microwave Conference-European Wireless 99, Munich, Germany, 1999, pp. 13-16.

[26] Y. Okumura, E. Ohmori, T. Kawano, and K. Fukuda, "Field strength and its variability in VHF and UHF land-mobile radio service," Rev. Elec. Commun. Lab., vol. 16, no. 9-10, pp. 825-873, 1968.

[27] K. Rizk, J. F. Wagen, and F. Gardiol, "Two-dimensional ray tracing modeling for propagation prediction in microcellular environmnents," IEEE Trans. Veh. Technol., vol. 46, pp. 508-517, 1997.

[28] K. R. Schaubach, N. J. Davis, and T. S. Rappaport, "A ray tracing method for predicting path loss and delay spread in microcellular environments," in Proc. Vehicular Technology Conference, 1992, pp. 932-935.

[29] S. Y. Tan and H. S. Tan, "A theory for propagation path loss characteristics in a city-street grid," IEEE Trans. Electromagn. Compat., vol. 37, pp. 333-342, 1995.

[30] J. H. Tarng and K. M. Ju, "A novel 3-D scattering model of 1.8-GHz radio propagation in microcellular urban environment," IEEE Trans. Electromagn. Compat., vol. 41, no. 2, pp. 100-106, 1999.

[31] L. L. Xie and P. R. Kumar, "A network information theory for wireless communication: Scaling laws and optimal operation," IEEE Trans. Inform. Theory, 2004, to be published. 


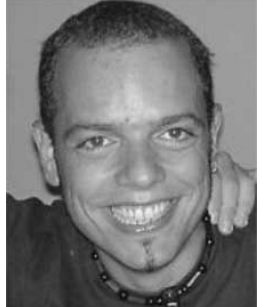

Massimo Franceschetti (M'03) received the Laurea degree (magna cum laude) in computer engineering from the University of Naples, Italy, in June 1997 and the M.Sc. and the Ph.D. degrees in electrical engineering from the California Institute of Technology, Pasadena, in 1999 and 2003, respectively.

During his studies, he spent one semester at the Department of Computer Science, University of Edinburgh, U.K., thanks to a fellowship by the Students Award Association of Scotland. He is currently a Postdoctoral Fellow at the University of California at Berkeley. His research interests include stochastic networks, wireless propagation models, applied probability, and distributed computing.

Dr. Franceschetti is a recipient of the 1999 UPE/IEEE Computer Society award for academic excellence, the 2000 Caltech Walker von-Brimer Foundation award for outstanding research initiative, and the 2003 Caltech Wilts prize for best thesis in electrical engineering.

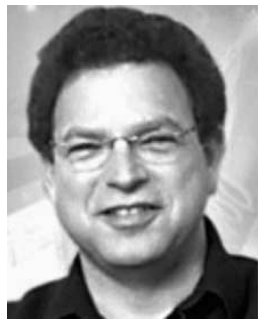

Jehoshua Bruck (S'86-M'89-SM'93-F'01) received the B.Sc. and M.Sc. degrees in electrical engineering from the Technion-Israel Institute of Technology, in 1982 and 1985, respectively, and the $\mathrm{Ph} . D$. degree in electrical engineering from Stanford University, Stanford, CA, in 1989.

$\mathrm{He}$ is the Moore Professor of Computation and Neural Systems and Electrical Engineering at the California Institute of Technology (Caltech), Pasadena. His research interests include paralle and distributed computing, fault-tolerant systems, error-correcting codes, computation theory and biological systems. His has an extensive industrial experience, including, with IBM for ten years both at the at the IBM Almaden Research Center and the IBM Haifa Science center. He is a co-founder and Chairman of Rainfinity, a spin-off company from Caltech that is focusing on providing software for high performance reliable Internet infrastructure. He published more than 150 journal and conference papers in his areas of interests and he holds 22 patents.

Dr. Bruck is the recipient of a 1997 IBM Partnership Award, a 1995 Sloan Research Fellowship, a 1994 National Science Foundation Young Investigator Award, six IBM Plateau Invention Achievement Awards, a 1992 IBM Out standing Innovation Award for his work on "Harmonic Analysis of Neural Networks" and a 1994 IBM Outstanding Technical Achievement Award for his contributions to the design and implementation of the SP-1, the first IBM scalable parallel computer.

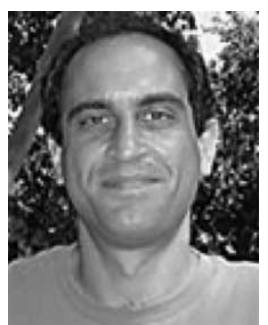

Leonard J. Schulman received the B.Sc. degree in mathematics in 1988 and the Ph.D. degree in applied mathematics in 1992, both from the Massachusetts Institute of Technology (MIT), Cambridge.

Since 2000, he has been an Associate Professor of Computer Science at the California Institute of Technology, Pasadena. He has held appointments as NSF mathematical sciences Postdoctoral Fellow at the University of California, Berkeley, visiting scientist at the Weizmann Institute of Science, assistant and associate professor at the Georgia Institute of Technology, and visiting member at the Mathematical Sciences Research Institute.

Dr. Schulman is a recipient of the Jon A. Bucsela prize in mathematics at MIT and an NSF CAREER award. 